\title{
New developments in the treatment of early-onset spinal deformity: role of the Shilla growth guidance system
}

This article was published in the following Dove Press journal:

Medical Devices: Evidence and Research

20 July 2016

Number of times this article has been viewed

\author{
Sean M Morell' \\ Richard E McCarthy ${ }^{2}$ \\ 'Department of Orthopaedics, \\ University of Arkansas for \\ Medical Sciences, ${ }^{2}$ Department of \\ Orthopaedics, Arkansas Children's \\ Hospital, Little Rock, AR, USA
}

Correspondence: Richard E McCarthy Department of Orthopaedics, Arkansas Children's Hospital, I Children's Way, Slot 839, Little Rock, AR 72202, USA

$\mathrm{Tel}+\mid$ 50| 364 |42।

Fax + I 50I 364 I522

Email Rmccarthy@uams.edu

\begin{abstract}
Early-onset scoliosis is a complex condition with multiple facets. The goal of treating any spinal deformity is to improve the condition of the patient with the least intervention necessary. A system that allows for continuation of natural spinal growth while correcting the deformity should be the goal of treating this complex condition. The SHILLA growth guidance system allows for continued growth of the pediatric spine while correcting and guiding the apex and guiding the future growth of the curvature. The system involves selective fusion across the apex of the curvature, and minimally invasive instrumentation is then used above and below the apex to allow for continued growth of the spine. A review of recent literature on the SHILLA growth guidance system shows promising results. Early animal models showed continued growth across unfused levels with minimal facet articular damage. Comparative studies to traditional growing rods showed significantly less total surgeries along with comparable correction and longitudinal growth. The SHILLA growth guidance system is a good option for this complex patient group. Results are comparable with other growing constructs with significantly less operative interventions. The SHILLA system allows for natural growth of the pediatric spine while correcting the scoliotic deformity in a minimally invasive method. The goal of this article is to present a comprehensive review of the SHILLA system surgical technique and the associated literature concerning this topic.
\end{abstract}

Keywords: scoliosis, SHILLA, growing, early-onset, growth friendly

\section{Introduction}

Early-onset spinal (EOS) deformities present many challenges. There is a wide array of etiologies, associated syndromes, and variable manifestations of the deformities involved in the process that pose many problems with early treatment. ${ }^{1}$ The main goal of treating any deformity in the growing skeleton is to allow for the skeleton to continue growing unimpeded. Spinal growth is intricately related to healthy cardiac and respiratory development by providing for appropriate thoracic development. In these patients, impaired thoracic volume is closely related to morbidity and mortality. ${ }^{2}$ Therefore, the goal of any corrective surgery is to allow for satisfactory correction of the deformity while allowing for continued spinal growth.

There are many modalities for the treatment of EOS deformities. The use of single and dual growing rod constructs is based on providing a stable construct spanning over the shortest number of spinal segments to allow for growth but stop the progression of the deformity. This method does involve subsequent revisits to the operative suite for lengthening every 6-12 months. The vertical, expandable prosthetic titanium rib 
(VEPTR; Johnson \& Johnson, New Brunswick, NJ, USA) directly addresses the chest wall component of EOS by expanding the thoracic capacity for space. It does this through osteotomies or intercostal muscle lysis to enlarge the thoracic cavity directly. This system also requires return trips to the operating suite for expansion every $6-12$ months. ${ }^{1}$ Hybrid systems utilize a hook-rib anchoring system like that of VEPTR and can also attach to the vertebral column. The SHILLA growth guidance technique (Medtronic, Dublin, Ireland) attempts to harness the child's natural growing spine to achieve the appropriate adult height without the need for return to the operating suite for scheduled lengthening. ${ }^{3}$ Fiveyear follow-up on the patients treated with SHILLA have shown continued growth with promising results. The goal of this article is to present a concise review of the SHILLA system technique and the results of several pertinent studies.

\section{Materials and methods}

\section{SHILLA growth guidance technique}

SHILLA is based on correcting the apex of the deformity while allowing for the remaining spinal column to continue in its natural pattern and rate of growth. By focusing only on the apex of the curvature, the rest of the spinal column is spared from damage to apophyseal growth, which is necessary for longitudinal growth.

\section{Preoperative planning}

SHILLA is intended for use in pediatric patients with severe, progressive, and life-threatening scoliosis before the age of 10 years. Patients are selected for this procedure based upon the size of the curvature $\left(>50^{\circ}\right)$, the amount of growth remaining, health status for undergoing a 4-5 hour procedure, and careful discussion with parents concerning the risks/benefits of undergoing this procedure. Preoperative planning is key to the success of this procedure. Preoperative blueprinting of the surgical procedure along with coordination of both anesthesia and the surgical team are imperative for the success of this procedure. ${ }^{4}$ Children with EOS often have other comorbidities along with underlying cardiopulmonary issues that can impact preoperative planning and can potentially be benefited by the procedure.

Preoperative full- spine X-rays with flexibility films help to assess the correctability of the spinal deformity. The least derotated vertebrae on the bend films identify the inciting apical deformity that requires correction to neutral in all planes. Pelvic obliquity can be identified and appropriately planned for at this time also. Preoperative screening also includes magnetic resonance imaging of the full spine in those less than 10 years old to rule out intrathecal abnormalities. Assessment of pulmonary status is also necessary due to length and stress of surgery. ${ }^{4}$ This can be done with pulmonary function testing (PFT) if the child can perform the study, but otherwise the child is closely evaluated by a pediatric pulmonologist. It is also necessary to determine potential growth. Wrist and hand films are sometimes obtained to determine bone age and assess potential growth. ${ }^{5}$

\section{Surgical procedure}

The procedure is performed in a single midline incision along the length of the entire construct. Using intraoperative fluoroscopy, the apical segments that are to be addressed based on preoperative planning are identified and marked with sterile needles. These segments are the only ones to be dissected in this procedure. A subperiosteal dissection is utilized to encourage fusion across the apex. Appropriate osteotomies (Ponte, pedicle subtraction, and vertebral column resection) are conducted at these levels to provide for appropriate correction in all planes. In some cases, it is necessary to utilize intraoperative traction of the spine. Our preferred method of traction is Gardner-Wells tongs and distal femoral traction. Fixed head or uniaxial screws are used at the apex to maximize the correction during the derotation maneuver. The apex of the deformity, previously identified based on preoperative imaging, is the only portion of the spine that is treated with these fixed or uniaxial pedicle screws. The standard spinal system contains instruments that attach to the pedicle screws to allow for a derotational maneuver. Growing screws are placed above and below the apex deformity and fusion segment. ${ }^{5}$

The growing screws are placed so as to preserve the growth potential by avoiding the subperiosteal approach. The paraspinal musculature fascia is incised linearly $1 \mathrm{~cm}$ on each side of the spinous processes above and below the apex. Through the musculature, Jamshidi needles under direct fluoroscopy are used to place polyaxial cannulated pedicle screws at levels predetermined to control the compensatory curves. Initial corrective maneuvers are applied to the apical segments using a provisional convex rod with sagittal derotation and coronal realigning maneuvers are conducted. Once the final coronal and sagittal correction is obtained, a strong derotation maneuver is applied to the apex to correct the axial plane deformity. The final position is held with a cross-link to prevent rod rotation.

Either $4.5 \mathrm{~mm}$ (weight $<30 \mathrm{~kg}$ ) or $5.5 \mathrm{~mm}$ (weight $>30 \mathrm{~kg}$ ) rods are used. Standard locking caps are placed at the apex to firmly hold the rods to the spine. SHILLA caps, which tighten to the screw heads, are placed in all the growing screws. By 
not engaging to the rod, these caps allow for guided growth in both the sagittal and coronal planes while allowing for longitudinal growth along the rods. The rods are left long, $2 \mathrm{~cm}$ protruding beyond the screws at each end. ${ }^{5}$

Closure brings fascial layers over the top of the implants. An intraoperatively fit thoraco-lumbo-sacral orthosis is worn for 3 months postoperatively. ${ }^{5}$

\section{The instrumentation}

The SHILLA growth guidance system uses both standard pedicle screw and posterior spinal fusion techniques along with specially designed cannulated polyaxial pedicle screws (SHILLA growth guidance screws) (Figure 1) and nonlocking caps (Figure 2).

Fixed or monoaxial pedicle screw fixation (Figure 3) is only used at the apex of the curvature to maximize derotational correction. The placement of these screws involves a subperiosteal dissection and disruption of the potential growth at these levels. Both sides of the apex are instrumented to allow for firm control and maximum correction of the

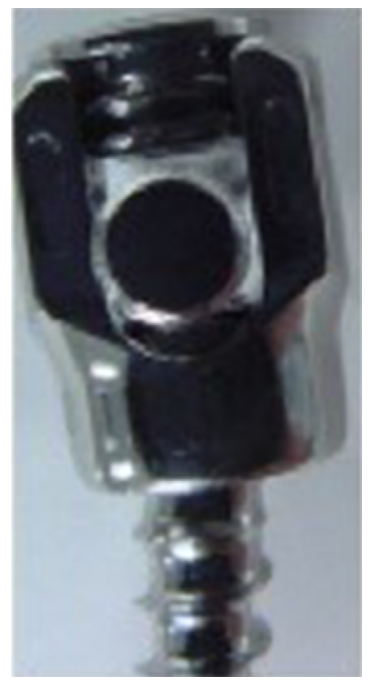

Figure I The Shilla screw with cap and rod in final position.

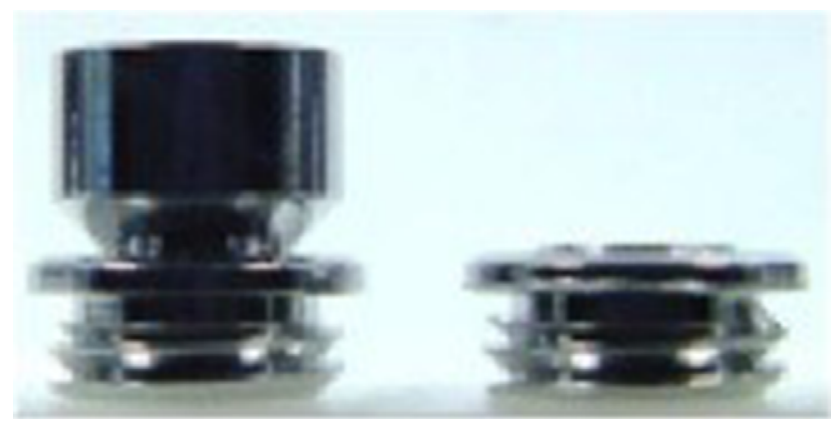

Figure 2 The Shilla cap.

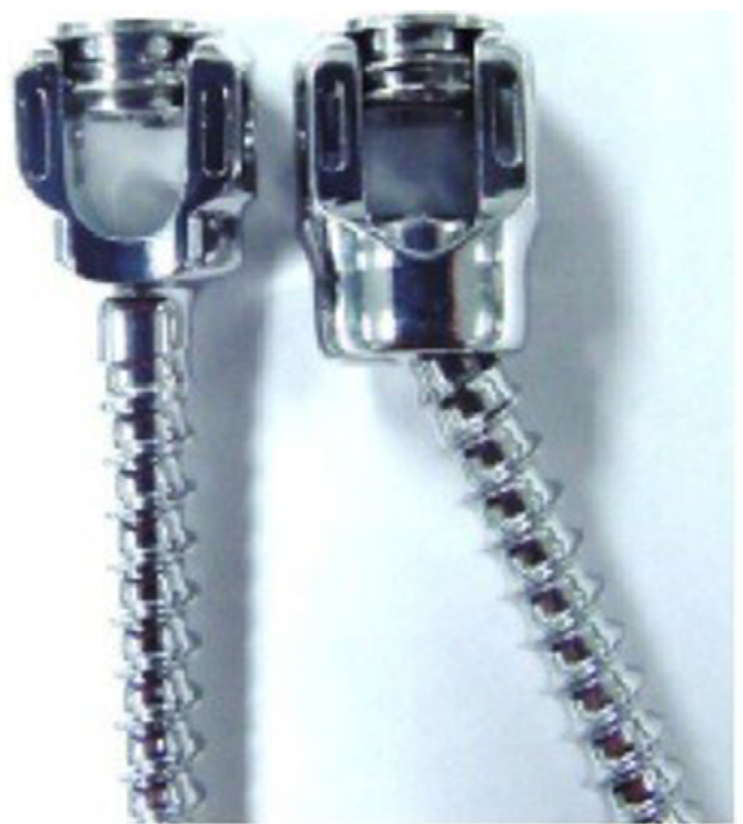

Figure 3 An example of the Shilla screw with cap. Note: This configuration allows for the rod to slide freely.

curvature as well as supplying a force sufficient to overcome the tendency for the abnormal growth known as crankshaft. Screw size is estimated on preoperative blueprints and modified based upon intraoperative fluoroscopy. Traditional locking screw caps are then used to hold the rod in place.

SHILLA growth guidance screws are cannulated polyaxial pedicle screws (Figure 3) placed using a minimally invasive method and are essential to preserving the potential growth at these levels. Using a Jamshidi needle under direct fluoroscopic visualization, the pedicle is found and the needle is placed in the appropriate position. Using the Jamshidi needle as a guide, the SHILLA cannulated pedicle screw is placed appropriately and checked under fluoroscopy. Caudal to the apical curvature, a bilateral pedicle screw pattern is usually placed. Cephalad to the apical curvature, an alternating pedicle screw pattern is usually placed. The SHILLA caps allow the rods to slide longitudinally allowing for growth while imparting stability in the sagittal and coronal planes.

A low-profile cross-link caudal to the curvature is often utilized for rotational stability. ${ }^{5}$ Also, between the apical segments and the growing segments, specially made "C-clamps" are used to prevent the rods from sliding distally should they break. These are essentially clamps on the rods to prevent the rods from sliding out of the construct in the case of rod failure.

\section{Results and discussion}

The SHILLA system was initially tested on a caprine model using eleven goats with SHILLA instrumentation over 6 
months. The study was designed to confirm growth of the spine and check for spinal stenosis. At 6-month follow-up, an average longitudinal growth of $48 \mathrm{~mm}$ (37-62 $\mathrm{mm})$ was confirmed in the model, and there was no evidence of stenosis with either the bilateral screw or the alternating screw pattern. Micro-computed tomography was used to evaluate the facet joints at the multiple levels of the SHILLA system. The transitional zone between fusion and growing levels showed signs of degeneration in the facets. The levels between the transitional zone and the growing levels were found to have normal articular cartilage. Facets at the level of the SHILLA screws did show degenerative changes thought to be due to the screw abutment against the pedicle and superior process of the facet. The instrumentation itself showed wear at the interval of the rod and the SHILLA screws from movement (Figure 4). These wears did not cause any structural failures and manual testing showed free motion across this interval after dissection. Metallic wear and debris were observed adjacent to the SHILLA screws and were also seen in adjacent para-aortic lymph nodes. The lymph nodes appeared dark and microscopic evaluation revealed metallic debris in the lymphoid cells. Only the nodes near the SHILLA screws manifested these changes. Metallic debris was found to be up to $45 \mu \mathrm{m}$ in size. ${ }^{6}$ This metallic tissue staining has also been noted in the human patient population and has not caused any adverse effects to date (Figure 5).

In a study comparing 36 patients treated with the SHILLA growth guidance system and 36 patients treated with traditional distraction growing rods, the growing rod group showed an improvement in Cobb angle and an increase in T1S1 length compared to the SHILLA group. In spite of this, growing rod systems did require significantly more number of surgeries. There was no difference in the complication rate between the two groups. The average final improvement in Cobb angle in the growing rod group was found to be $36^{\circ}$, and the average improvement in the SHILLA group was found to be $23^{\circ}$. Although the initial Cobb angle improvement was greater in the SHILLA group, over time the Cobb angles increased in the SHILLA group. The average total spinal height in the growing rod group was $8.8 \mathrm{~cm}$ while in the SHILLA group was $6.4 \mathrm{~cm}$. SHILLA group patients had an average of 2.8 surgeries compared to the growing rod group averaging 7.4 surgeries. ${ }^{7}$

A recent retrospective review of 33 patients with a 5-year minimum follow-up on patients treated with SHILLA instrumentation for early-onset scoliosis (neuromuscular, idiopathic, congenital, syndromic) showed promising results. The average follow-up on these patients was 7 years

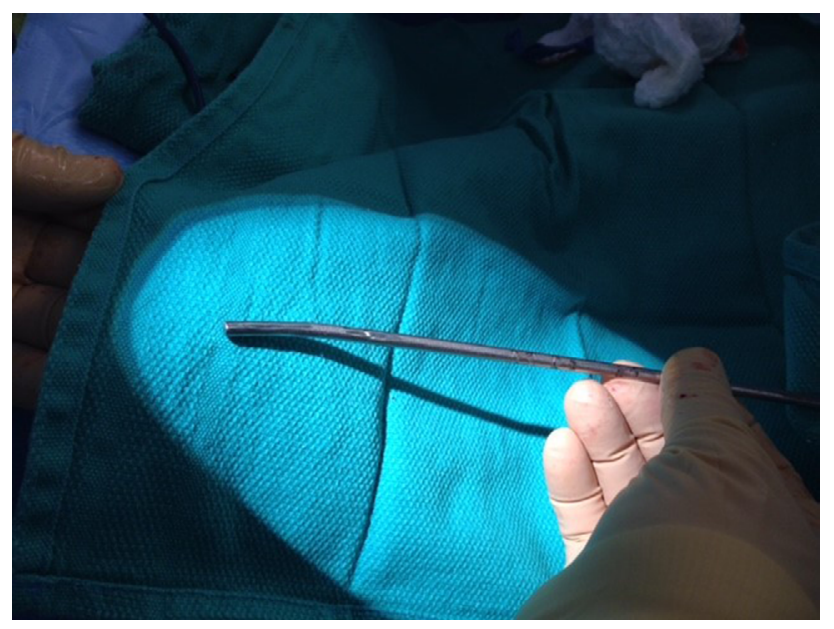

Figure 4 This image shows in situ wear on the rod from the Shilla screw.

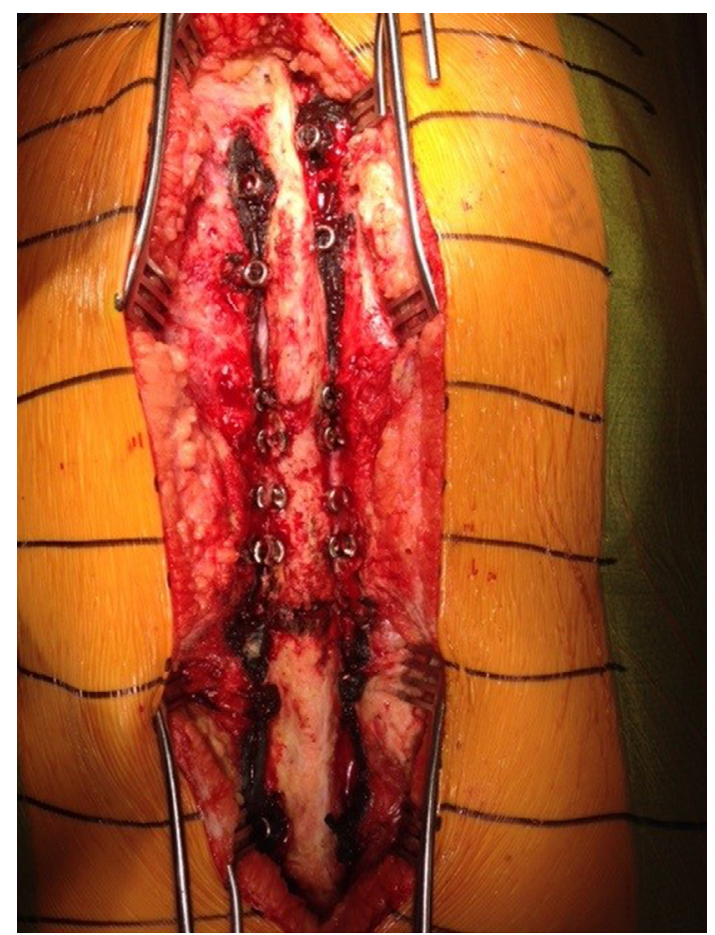

Figure $\mathbf{5}$ In situ metallosis in patients.

Note: In situ metallosis has been seen in many patients and has not shown any adverse effects on the individuals.

(minimum of 5-year follow-up). The average preoperative curves measured $69^{\circ}$, and the average curves at the most recent follow-up were found to be $44^{\circ}$. Complications (secondary infections, alignment issues, implant-related complications) were also investigated in this study. ${ }^{8}$

As previously discussed, the maintenance of thoracic spinal growth and thoracic volume is the goal of any growing system. Using the SHILLA system, the space available for lungs increased by an average of $27.7 \%$ in the right and $30.5 \%$ in the left (measured using the Campbell technique). 
Limitations imposed by age and mental status make PFTs impractical in most of these patients, but for this study, six patients of the 18 who had preoperative pulmonary issues were able to complete PFT at spinal maturity. Of the six, all but one patient had forced vital capacity and forced expiratory volume in 1 second values $>50 \%$ at the end of the study. The one patient discussed had spinal muscular atrophy (associated pulmonary deficits are expected in such patients). ${ }^{8}$

Complications include wound breakdown, spinal alignment issues, proximal junctional kyphosis, implant-related complications, and metallosis. Six patients had complications with wound breakdown requiring a return to the operating room. One developmentally delayed patient had wound healing issues due to frequently scratching the wound. The remaining patients had wound complications due to problems with prominent implants. ${ }^{8}$

Infection in four patients required implant removal with reimplantation in three after 6 months. Preoperative optimization of patients' nutrition is an important determinant in the prevention of wound complications. Many of these children have multiple comorbidities that preclude them to malnutrition, making nutritional optimization prior to surgery very difficult. ${ }^{8}$

Spinal alignment issues were seen in eight patients: six had progression of the curve $\left(>15^{\circ}\right)$ and two had progression of pelvic obliquity. Five of the eight were skeletally mature enough to undergo a definitive fusion. One underwent extension of the instrumentation to the pelvis, and one underwent replacement with dual growing rods (patient had a double major curve). One underwent correction of his curvature with SHILLA implant revision. ${ }^{8}$

Proximal junctional kyphosis is a phenomenon sometimes seen in the postoperative period in patients who have undergone spinal fusions. It involves a dramatic increase in kyphosis proximal to the instrumented levels due to acute change in rigidity between the fused spine and the unfused spine. Proximal junctional kyphosis is measured from the upper endplate above the upper-instrumented level to the lower end plate below the upper-instrumented level and was found in three patients. Two of the three patients had not yet undergone their definitive fusion.

Twenty-four patients had implant-related complications: screw pullout/loosening (nine), broken cables (one), rod prominence (14), growth off of rods (five), and rod fracture (18). Eight of the rod fractures occurred in patients in whom transition rods from 4.5 to $3.5 \mathrm{~mm}$ were used for added flexibility. Most fractures occurred at the junctions between these transitions. In most active children, $3.5 \mathrm{~mm}$ rods failed before 2 years and $4.5 \mathrm{~mm}$ rods failed by 5 years. There have been no fractures in $5.5 \mathrm{~mm}$ rods to date. ${ }^{8}$

\section{Conclusion}

In conclusion, the SHILLA growth guidance system offers an innovative natural growing option for treating EOS deformities. Early results are comparable with other systems with significantly fewer operative procedures for SHILLA patients. In a patient group that has many associated comorbidities, any decrease in the number of anesthetics is significant. By addressing primarily the apex of the scoliotic curvature and guiding growth of the compensatory curves, this procedure is far less invasive and the child is allowed to grow in a natural manner. Early complications are decreasing in frequency with experience and improvement in techniques.

\section{Disclosure}

Sean Morell, MD, has no conflicts of interest to disclose in this work. Richard McCarthy, MD, is a consultant and member of the Speaker's Bureau for Medtronics and receives royalties from his work with them.

\section{References}

1. Thompson GH, Lenke LG, Akbarnia BA, McCarthy RE, Campbell RM, Jr. Early onset scoliosis: future directions. J Bone Joint Surg Am. 2007:89(Suppl 1):163-166.

2. Williams BA, Matsumoto H, McCalla DJ, et al. Development and initial validation of the Classification of Early-Onset Scoliosis (C-EOS). J Bone Joint Surg Am. 2014;96(16):1359-1367.

3. Gupta MC, Gupta S, Vaccaro AR. Complex Spine Cases: A Collection of Current Techniques. New Delhi: Jaypee Brothers Medical (P); 2015.

4. McCarthy RE, Luhmann SJ. Growth-Guided Instrumentation: Shilla Procedure In: Akbaria BA, Yazici M, and Thompson GH, editors. The Growing Spine, Management of Spinal Disorders in Young Children. 2nd ed. Springer; 2016:701-711.

5. McCarthy RE. Shilla. In: Skaggs DL, Kocher MS, editors. Master Techniques in Orthopaedic Surgery, Pediatrics. 2nd ed. Wolters Kluwer; Wolters Kluwer; 2016:607-616.

6. McCarthy RE, Sucato D, Turner JL, Zhang H, Henson MA, McCarthy $\mathrm{K}$. Shilla growing rods in a caprine animal model: a pilot study. Clin Orthop Relat Res. 2010;468:705-710.

7. Andras LM, Joiner ERA, McCarthy RE, et al. Growing rods versus Shilla growth guidance: better cobb angle correction and T1-S1 length increase but more surgeries. Spine Deform. 2015;3(3):246-252.

8. McCarthy RE, McCullough FL. Shilla growth guidance for early onset scoliosis: results after a minimum of five years of follow-up. J Bone Joint Surg Am. 2015;97(19):1578-2884. 


\section{Publish your work in this journal}

Medical Devices: Evidence and Research is an international, peerreviewed, open access journal that focuses on the evidence, technology, research, and expert opinion supporting the use and application of medical devices in the diagnosis, monitoring, treatment and management of clinical conditions and physiological processes. The identification of novel devices and optimal use of existing devices which will lead to improved clinical outcomes and more effective patient management and safety is a key feature. The manuscript management system is completely online and includes a quick and fair peer-review system. Visit http://www. dovepress.com/testimonials.php to read real quotes from authors.

Submit your manuscript here: https://www.dovepress.com/medical-devices-evidence-and-research-journal 